\title{
Vortex fluidic exfoliation of graphite and boron nitride
}

\author{
Xianjue Chen, ${ }^{\mathrm{a}}$ John F. Dobson, ${ }^{\mathrm{b}}$ and Colin L. Raston ${ }^{\mathrm{a}, *}$ \\ Received (in XXX, XXX) Xth XXXXXXXXX 20XX, Accepted Xth XXXXXXXXX 20XX \\ DOI: $10.1039 / b 000000 x$
}

\begin{abstract}
Graphite is exfoliated into graphene sheets by the shearing in vortex fluidic films of N-methyl-pyrrolidone (NMP), as a controlled process for preparing oxide free graphene with minimal defects, and for the exfoliation of the corresponding boron nitride sheets.
\end{abstract}

${ }_{10}$ Solution based methods have been widely used for the synthesis of graphene from graphite or graphite oxide, ${ }^{1}$ using high energy sonication for the exfoliation process in generating mono- or multi-layer structures. ${ }^{2-8}$ However, the associated cavitation process can result in damage to the graphene, ${ }^{2}$ which can affect 15 its properties. ${ }^{9,10}$ Developing facile methods for accessing viable quantities of graphene devoid of such defects, and also of chemical degradation, is therefore important in advancing the myriad of applications of graphene. To this end we have developed a shearing process for exfoliating graphite in an 20 organic solvent, namely NMP (N-methyl-pyrrolidone). The shearing involves vortex fluidics in a rapidly rotating tube, and is an alternative and tunable energy source for the exfoliation process with potentially minimal damage of the graphene. Sonication induced exfoliation of graphite, and other laminar 25 materials, occurs in $\mathrm{NMP}^{2,3,11}$ which has similar surface tension relative to graphene, and acts as a stabilising surfactant to avoid reassembling/restacking of the graphene. ${ }^{2}$ The exfoliation process establishes a new paradigm in the 'top down' fabrication of nanomaterials in solution, and we also demonstrate its versatility in 30 using the process to exfoliate iso-structural boron nitride $(\mathrm{BN})$.

The exfoliation occurs in the thin film in a vortex fluidic device (VFD) which has a rapidly rotating $10 \mathrm{~mm}$ glass tube inclined at $45^{\circ}$, Figure 1. Shearing in the film arises from the interplay between centrifugal and gravitational forces, the thin film being 35 parabolic in shape, although at high speed this approximates to having a thin film along the tube. We have previously used such shearing in the VFD to disassemble molecular capsules based on two $p$-phosphonated calix[5] arenes held together by a seam of hydrogen bonds, for the encapsulation of the drug molecule 40 carboplatin. ${ }^{12}$ An important feature of the thin film is that it is maintained for a fixed volume of liquid within the tube (confined mode), which overcomes the need for using large volumes of liquids for shearing processes associated with conventional continuous film flow microfluidic platforms, notably spinning ${ }_{45}$ disc processors (SDP) ${ }^{13,14}$ and rotating tube processors (RTP). ${ }^{14-16}$ Nevertheless, the shearing in SDP thin films is effective in controlling the disassembly of molecular capsules based on six hydrogen bonded pyrogall[4] arenes molecules. ${ }^{13}$

In a typical experiment graphene sheets were prepared from a 50 suspension of pristine graphite in NMP $(1 \mathrm{~mL}$, particle size $7-10$ $\mu \mathrm{m}$ in lineal dimension of the planar flakes) with the inclined tube rotating at $7000 \mathrm{rpm}$ for $30 \mathrm{~min} .^{*}$ The yield of the monolayer graphene sheets was approximately $1 \mathrm{wt} \%$. We note that the
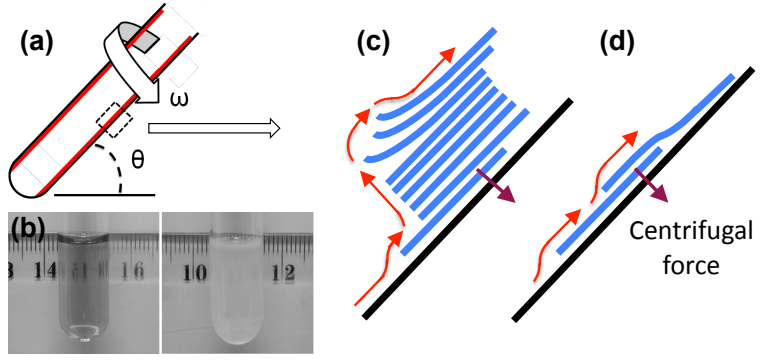

Figure 1. (a) Schematic of the vortex fluidic device $(10 \mathrm{~mm}$ diameter tube, $16 \mathrm{~cm}$ long, inclined at $45^{\circ}$, operating at 7000 and $8000 \mathrm{rpm}$ for graphite and BN respectively). (b) Photographs of the resulting colloidal suspensions of graphene (left) and BN (right) in NMP. (c) Carton of the exfoliation process, and (d) slippage on the inner surface of the rotating tube, with the red arrows indicating the direction of the shearing flow.

thickness of the graphite flakes are significantly decreased for ${ }_{55}$ rotating speeds larger than $5000 \mathrm{rpm}$. A systematic evaluation of the effect of different inclination angles $\left(0\right.$ to $\left.90^{\circ}\right)$ and rotational speeds (3000-9000 rpm) established the optimised speed for exfoliation at $7000 \mathrm{rpm}$ for the tube inclined of the order $45^{\circ}$ and slightly more. Clearly the angle and speed affects the 60 shearing/exfoliation, although there would be an upper limit of the speed where the centrifugal force accelerates the flat graphite flakes and any generated graphene to the surface of the tube, possibly minimising the extent of exfoliation. Indeed it may result in restacking of any graphene sheets.

${ }_{65}$ TEM (transmission electron microscope) images of the graphene established the formation of mono-layer and few-layer material, Figures $2 \mathrm{a}$ and $2 \mathrm{~b}$, with the maximum size of sheets $c a$ $2 \mu \mathrm{m}$ in cross section. This is less than that of the original

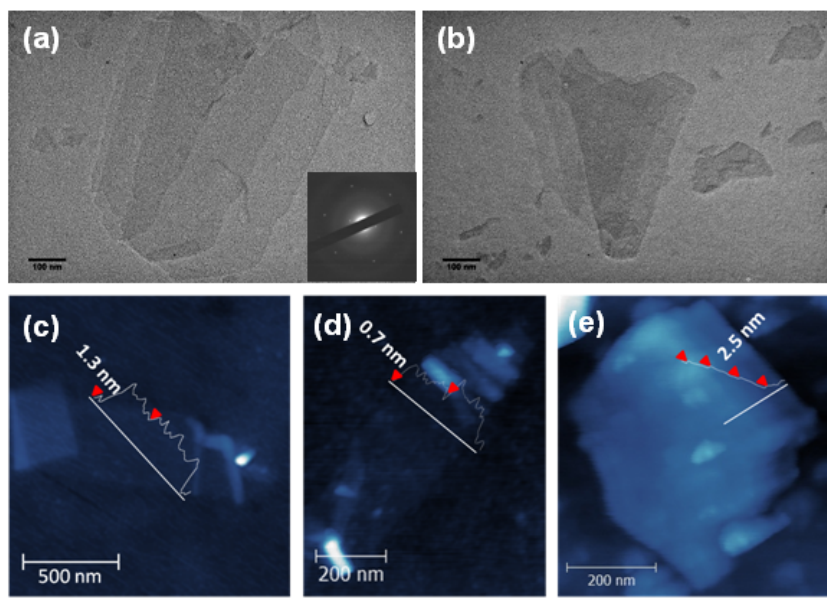

Figure 2. (a) and (b) TEM of exfoliated graphene sheets, with an SAED pattern inset for (a). (c) - (e) AFM images of graphene sheets. 
graphite flakes, ca $7-10 \mu \mathrm{m}$ in cross section, and suggests that the smaller graphite flakes are easier to exfoliate than the larger ones, and this is consistent with the smaller collective van der Waals interactions between layers for smaller graphene sheets. 5 Additional TEM images are shown in Figure S1, ESI.

Selected area electron diffraction (SAED), Figure 2a inset, for the complete area in the TEM image, has the expected hexagonal distribution of peaks for pristine graphene sheets. Height profile measurements using atomic force microscopy (AFM), Figure $2 \mathrm{c}$

10 and $2 \mathrm{~d}$, were close to $1 \mathrm{~nm}$, in accordance with mono-layer graphene sheets. ${ }^{2}$ An AFM height scan through the edge of a fewlayer graphene sheet, Figure 2e, reveals a step-wise structure, which is consistent with the exfoliation process.

The formation of graphene arises from the shearing within the 15 thin film which overcomes the large collective van der Waals interactions between the graphene layers, with the NMP acting as both a solution medium for the exfoliating process and a stabilizer to prevent the graphene sheets from reassembling/restacking. The graphene is conveniently prepared 20 by this method, with no evidence of extensive damage/defects, and without the need for oxidising the graphite prior to the processing, unlike for other solution based exfoliation methods. ${ }^{5-7}$

Boron nitride $(\mathrm{BN})$ flakes were also exfoliated using the same process, with $8000 \mathrm{rpm}$ for the tube inclined of the order $45^{\circ}$ or 25 slightly more giving the highest yield, estimated at $\leq 10 \%$. TEM images revealed monolayer sheets, Figure 3a. The SAED pattern in Figure 3c was obtained for the boron nitride sheet in Figure 3a, and shows the expected six-fold symmetry. The mono- and multilayers are identified by analysing the ratio of the intensity of the $30\{1100\}$ peaks relative to the $\{2110\}$ peaks, with $\mathrm{I}_{\{1100\}} / \mathrm{I}_{\{2110\}}>1$, for mono-layers, and $<1$ for multi-layers with Bernal (AB) stacking. The intensity scan along the dashed line in Figure $3 \mathrm{c}$ shows that the $\{1100\}$ peaks are more intense than the $\{2110\}$ peaks, confirming mono-layer BN in Figure 3a.
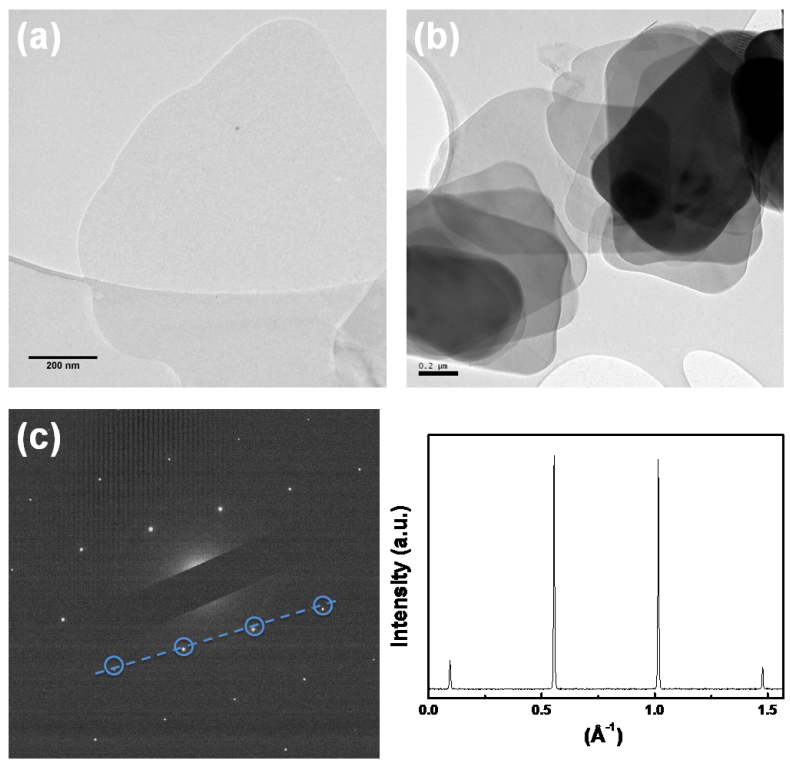

Figure 3. (a) TEM of a single sheet of BN. (b) TEM of adjacent BN layers with the same size and shape. (c) SAED pattern of the boron nitride sheet shown in (a) and the intensity scan along the dashed line.

35 Figure $3 \mathrm{~b}$ highlights $\mathrm{BN}$ sheets with the same outline ("finger print'), which suggests that they originate from the same flake of the bulk material, which is similar to the finding for graphene, Figure 2a. It is noteworthy that the BN precursor used in the exfoliation experiments has a smaller average particle size
40 diameter than the graphite flakes, ca 2-3 $\mu \mathrm{m}$ versus ca $7-10 \mu \mathrm{m}$. The smaller size appears to favour a greater amount of exfoliation, ca $10 \%$ cf ca $1 \%$ for graphite, which is consistent with relative surface area stacking energies. The partially exfoliated BN and graphene are presumably stabilised by the 45 solvent and 'frozen' as a slipped structure. Element mapping of the BN mono-layer in Figure 3a, and additional TEM images of the $\mathrm{BN}$ "finger print" are shown in Figure S2, ESI. Both graphene and $\mathrm{BN}$ sheets were also characterised using scanning electron microscopy (SEM), Figure 4.

s0 The aforementioned 'finger print' of partially stacked graphene and $\mathrm{BN}$ sheets clearly highlights the effectiveness of the shearing thin film conditions in exfoliating the laminar materials, and gives insight into the mechanism of the process. The displacement of single layers of graphene or BN relative to each 55 other suggests that the exfoliation involves a slippage process, which is consistent with the limited directional aspect of the van der Waals interactions between two sheets. However, such a slippage process would require the individual sheets to be partially lifted from the surface of the bulk material at some point ${ }_{60}$ to provide the necessary lateral force to start the slippage, Figure 1(c). An alternative or additional mechanism is that the exfoliation arises from a slippage of the graphene or BN directly on the walls of the tube, ie the flakes of the bulk material held against the tube wall by the centrifugal force experience a shear ${ }_{65}$ induced displacement along the tube, resulting in the graphene/BN exfoliating at the surface of the tube. This is consistent with the finding that some of the graphene is attached to the walls of the tube, although any exfoliated graphene/BN can be accelerated to the wall of the surface of the tube by the 70 centrifugal force. The partial lifting and slippage mechanism on the tube wall is distinctly different to the now famous use of scotch tape to exfoliate graphene. ${ }^{18}$

A more detailed understanding of the stability of slipped stacks of graphene sheets after the cessation of centrifugation may be 75 available as follows. To a first approximation in which the atomic 'graininess' of graphene is neglected, the stacking energetics in a slipped stack of graphene flakes is determined by the surface binding energy $\sigma$ per unit area at the equilibrium layer separation distance. For the contact of two graphene sheets, the binding so energy per in-contact atom can be estimated as about $1 / 2$ that for bulk graphite. From the current benchmark calculation ${ }^{19}$ the bulk graphite layer cohesive energy is $48 \mathrm{meV} /$ atom. This gives the graphene-graphene binding energy per unit area $\sigma_{\text {gg }}$ as about 0.17 $\mathrm{J} / \mathrm{m}^{2}$. For graphene-fluid contact the corresponding surface 85 energy will be denoted $\sigma_{\mathrm{gf}}$. In vacuo, only $\sigma_{\mathrm{gg}}$ is present, resulting in a relatively strong, approximately constant slippage force $\mathrm{F}_{0}=\sigma_{\mathrm{gg}} \mathrm{L}$ parallel to the sheets, tending to pull 'slipped' graphene sheets back into full registry (maximum overlap area). Here $\mathrm{L}$ is the projected length of the contact edge between the 90 slipped sheets. In the fluid environment, however, any graphenegraphene contact lost due to shearing away from perfect overlap is replaced by graphene-fluid contact so the restoring force is $\mathrm{F}_{0}=\left(\sigma_{\mathrm{gg}-} \sigma_{\mathrm{gf}}\right) \mathrm{L}$ which is presumably much weaker since, for this solvent, $\sigma_{\mathrm{gg}} \sim \sigma_{\mathrm{gf}}$. This means that a slipped stacking 95 configuration, once initiated by the fluidic shearing process, can be stabilized by the much weaker spatially periodic component $\mathrm{F}_{\text {osc }}$ of the slippage force, neglected above, that depends on registry of individual atoms between the two sheets. In fact the process of pulling back the upper sheet towards maximum 100 overlap with the lower sheet initially meets essentially no resistance, but, as more lines of $\mathrm{C}$ atoms overlap, the oscillatory component $\mathrm{F}_{\text {osc }}$ increases proportionally to the number of overlapped lines of carbon atoms while the basic retraction force 
$\mathrm{F}_{0}$ remains constant as discussed above. Thus, beyond a certain minimum number of overlapped lines, the total force $F_{0}+F_{\text {osc }}$ vanishes near each $\mathrm{AB}$ stacking configuration. This provides a series of potential wells of increasing depth as the sheets are pulled together by $\mathrm{F}_{0}$, and the sliding process can 'hang up' in one of these energy minima, preventing further increase of the overlap area. This suggests that stacks that were initially very strongly sheared may later come to equilibrium in the stationary fluid at a degree of overlap that is relatively independent of the 10 initial degree of shearing induced by the shearing flow. This semi-quantitative argument is supported by atomistic LennardJones simulations to be detailed elsewhere. This mechanism can explain the presence of stacks in the "slipped-deck-of-cards" configuration with well-defined but not complete overlap 15 between graphene flakes, as illustrated in Fig. 2a, in the solution after centrifugation has ceased. Furthermore, if the slipped graphene stack ends up adhering to the wall of the tube, further surface energies will come into play. Here the 'kinking' of the top graphene sheet at the edge of the next underlying sheet, in order 20 to achieve contact with the substrate, will bring strong sheetbending and edge cohesion forces into play, forces that may also

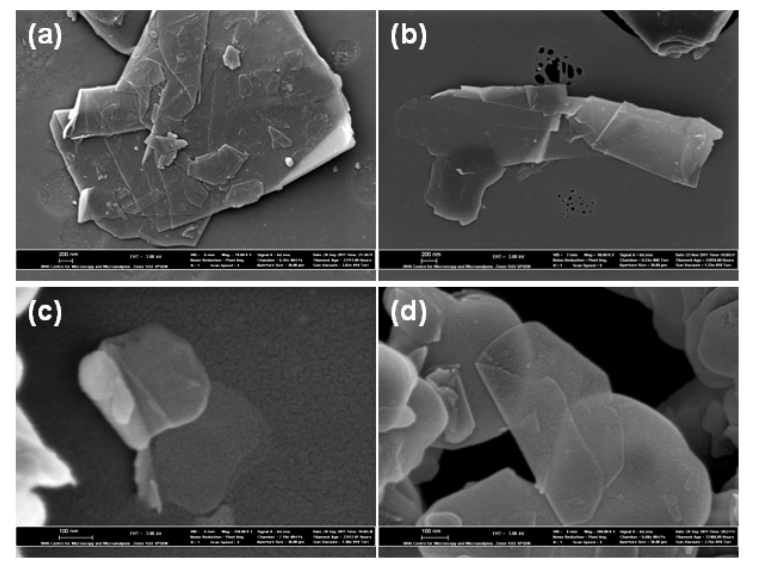

Figure 4. SEM images of (a) a graphene sheet adhered to the internal wall of a sacrificed glass tube, (b) exfoliated ultrathin graphene sheets, (c) 'finger print' of partially exfoliated BN sheets, and (d) folded BN sheets.

be able to stabilize the slipped configuration, Figure 1(d).

The use of a vortex fluidics device for a 'top down' fabrication of nano-materials is without precedent, and the method may be 25 applicable for the exfoliation of other laminar materials in a controlled way, with the sheets being relatively free of defects and chemical damage. The device provides a tunable 'soft energy' source in the form of shearing to exfoliate the laminar materials. This is in contrast to high energy wet ball milling ${ }^{17}$ or 30 high power sonication ${ }^{2,3}$ which can result in damage to graphene, which is one of the most promising materials for technology for the future, depending on its availability and purity.

We gratefully acknowledge the Australian Research Council, and the China Scholarship Council for support of this research, 35 and to Professors Mark Warner and Stuart Dalziel (Cambridge) for insight into the fluid dynamics. TEM and SEM work was carried out in the Centre for Microscopy, Characterisation and Analysis (The University of Western Australia). The authors also appreciate Ms Jing Zhao's support in the SEM studies.

\section{${ }_{40}$ Notes and references}

\footnotetext{
a Centre for Strategic Nano-Fabrication, School of Biomedical, Biomolecular and Chemical Sciences, The University of Western Australia, Crawley, WA 6009, Australia Fax: 618 6488 8683; Tel: 618 6488 3045; E-mail: colin.raston@uwa.edu.au
}

${ }_{45}^{b}$ Micro and Nanotechnology Centre, Griffith University, Nathan, Queensland 4111, Australia Fax: 6173735 7656; Tel: 6173735 7441; E-mail:j.dobson@griffith.edu.au

$\dagger$ Electronic Supplementary Information (ESI) available: [details of any supplementary information available should be included here]. See 50 DOI: $10.1039 / \mathrm{b} 000000 \mathrm{x} /$

+ Methods summary: Graphite flakes 7-10 $\mu \mathrm{m}$ in diameter were used as received from Alfa Aesar. Boron nitride flakes 2-3 $\mu \mathrm{m}$ in diameter were used as received from Sigma Aldrich. The graphite was suspended in Nmethyl-pyrrolidone (NMP) in targeting a $0.1 \mathrm{mg} / \mathrm{mL}$ concentration of 55 graphene. BN suspensions were similarly prepared targeting a $0.2 \mathrm{mg} / \mathrm{mL}$ of exfoliated material. The $10 \mathrm{~mm}$ glass tubes were washed with ethanol and acetone, and then air-dried prior to each experiment. The rotating speed was set at $7000 \mathrm{rpm}$ for graphite and $8000 \mathrm{rpm}$ for BN with the tube inclined at $45^{\circ}$. All experiments were for $1 \mathrm{~mL}$ of solution, for 30

60 minutes during, under ambient conditions. The as-treated suspensions were dropped onto 200 mesh holey carbon $\mathrm{Cu}$ grids for TEM characterization, and micrographs recorded using JEOL 2000FX and JEOL 2100 instruments operating at $80 \mathrm{keV}$ and $120 \mathrm{keV}$, respectively. SEM studies were carried out using Zeiss 1555 VP-FESEM with samples 65 coated with $3 \mathrm{~nm}$ Pt films. AFM images were recorded on Digital Instruments Dimension 3000 with a Nanoscope IIIa controller under tapping mode, using silicon cantilevers. Samples were drop cast onto a freshly cleaved mica and graphite substrate and excess solution removed using a gentle flow of nitrogen gas, also while spin coating. 70 .

1 J. Zhu, Nat. Nanotechnol., 2008, 3, 528

2 Y. Hernandez, V. Nicolosi, M. Lotya, F. M. Blighe, Z. Sun, S. De, I. T. McGovern, B. Holland, M. Byrne, Y. K. Gun'ko, J. J. Boland, P. Niraj, G. Duesberg, S. Krishnamurthy, R. Goodhue, J. Hutchison, V.

75 Scardaci, A. C. Ferrari and J. N. Coleman, Nat. Nanotechnol., 2008, 3, 563-568.

3 U. Khan, H. Porwal, A. O’Neill, K. Nawaz, P. May and J. N. Coleman, Langmuir, 2011, 27, 9077-9082.

4 X. Li, G. Zhang, X. Bai, X. Sun, X. Wang, E. Wang and H. Dai, Nat. Nanotechnol., 2008, 3, 538-542.

5 D. Li, M. B. Müller, S. Gilje, R. B. Kaner and G. G. Wallace, Nat. Nanotechnol., 2008, 3, 101-105.

6 V. C. Tung, M. J. Allen, Y. Yang and R. B. Kaner, Nat. Nanotechnol., 2009, 4, 25-29.

857 Y. Si and E. T. Samulski, Nano Lett., 2008, 8, 1679-1682.

8 C. J. Shih, A. Vijayaraghavan, R. Krishnan, R. Sharma, J. H. Han, M. H. Ham, Z. Jin, S. Lin, G. L.C. Paulus, N. F. Reuel, Q. H. Wang, D. Blankschtein and M. S. Strano, Nat. Nanotechnol., 2011, 6, 439-445.

9 F. Banhart, J. Kotakoski and A. V. Krashenlnnlkov, ACS Nano, 2011, 5, 26-41.

10 J. H. Chen, L. Li, W. G. Cullen, E. D. Williams and M. S. Fuhrer, Nat. Phys., 2011, 7, 535-538.

11 J. N. Coleman, M. Lotya, A. O’Neill, S. D. Bergin, P. J. King, U. Khan, K. Young, A. Gaucher, S. De, R. J. Smith, I. V. Shvets, S. K.

95 Arora, G. Stanton, H. Y. Kim, K. Lee, G. T. Kim, G. S. Duesberg, T. Hallam, J. J. Boland, J. J. Wang, J. F. Donegan, J. C. Grunlan, G. Moriarty, A. Shmeliov, R. J. Nicholls, J. M. Perkins, E. M. Grieveson, K. Theuwissen, D. W. McComb, P. D. Nellist and V. Nicolosi, Science, 2011, 331, 568-571.

10012 A. D. Martin, R. A. Boulos, L. J. Hubble, K. J. Hartlieb and C. L. Raston, Chem. Commun, 2011, 47, 7353-7355.

13 K. S. Iyer, M. Norret, S. J. Dalgarno, J. L. Atwood and C. L. Raston, Angew. Chem. Int. Ed., 2008, 47, 6362-6366.

14 J. Fang, Y. Guo, G. Lu, C. L. Raston and K. S. Iyer, Green Chem., 2011, 13, 817-819.

15 J. Fang, Y. Guo, G. Lu and C. L. Raston, Dalton Trans., 2011, 40, 3122-3124.

16 S. Dev, K. S. Iyer and C. L. Raston, Lab Chip, 2011, 11, 3214-3217.

17 W. Zhao, M. Fang, F. Wu, H. Wu, L. Wang and G. Chen, J. Mater. Chem., 2010, 20, 5817-5819.

18 K. S. Novoselov, A. K. Geim, S. V. Morozov, D. Jiang, Y. Zhang, S. V. Dubonos, I. V. Grigorieva and A. A. Firsov, Science, 2004, 306, 666-669.

19 S. Lebegue, J. Harl, T. Gould, J. Angyan, G. Kresse and J. Dobson, Phys. Rev. Lett. 2010 105, 196401. 